\title{
A New Luminescence Beam Profile Monitor for Intense Proton and Heavy Ion Beams
}

\author{
T. Tsang, S. Bellavia, R. Connolly, D. Gassner, Y. Makdisi,
} T. Russo, P. Thieberger, D. Trbojevic, A. Zelenski

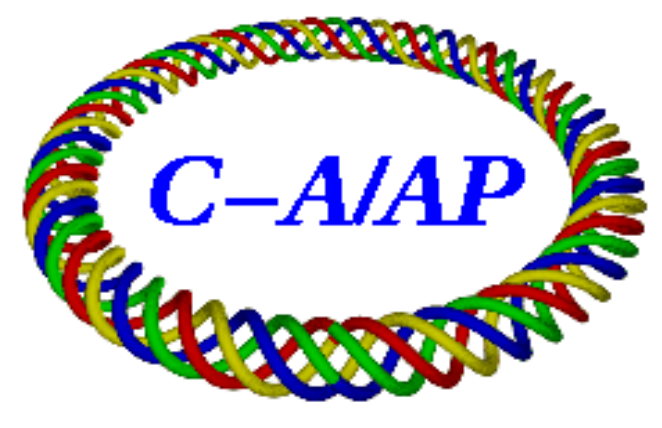

\section{Collider-Accelerator Department Brookhaven National Laboratory Upton, NY 11973}

Notice: This document has been authorized by employees of Brookhaven Science Associates, LLC under Contract No. DE-AC02-98CH10886 with the U.S. Department of Energy. The United States Government retains a nonexclusive, paid-up, irrevocable, world-wide license to publish or reproduce the published form of this document, or allow others to do so, for United States Government purposes. 


\title{
A new luminescence beam profile monitor for intense proton and heavy ion beams
}

\author{
T. Tsang, ${ }^{1}$ S. Bellavia, R. Connolly, D. Gassner, Y. Makdisi, T. Russo, P. Thieberger, \\ D. Trbojevic, and A. Zelenski \\ ${ }^{1}$ Instrtumentation Division, Brookhaven National Laboratory, Upton, NY 11953 \\ Collider Accelerator Division, Brookhaven National Laboratory, Upton, NY 11953
}

\begin{abstract}
A new luminescence beam profile monitor is realized in the polarized hydrogen gas jet target at the Relativistic Heavy Ion Collider (RHIC) facility. In addition to the spin polarization of the proton beam being routinely measured by the hydrogen gas jet, the luminescence produced by beam-hydrogen excitation leads to a strong Balmer series lines emission. A selected hydrogen Balmer line is spectrally filtered and imaged to produce the transverse RHIC proton beam shape with unprecedented details on the RHIC beam profile. Alternatively, when the passage of the high energy RHIC gold ion beam excited only the residual gas molecules in the beam path, sufficient ion beam induced luminescence is produced and the transverse gold ion beam profile is obtained. The measured transverse beam sizes and the calculated emittances provide an independent confirmation of the RHIC beam characteristics and to verify the emittance conservation along the RHIC accelerator. This optical beam diagnostic technique by making use of the beam induced fluorescence from injected or residual gas offers a truly noninvasive particle beam characterization, and provides a visual observation of proton and heavy ion beams. Combined with a longitudinal bunch measurement system, a 3-dimensional spatial particle beam profile can be reconstructed tomographically.
\end{abstract}

PACS numbers: 25.75.-q, 33.50.Dq, 41.75.-i, 29.25.it

Electronic mail: Tsang@bnl.gov 


\section{INTRODUCTION}

In some dedicated high energy physics experiments conducting at RHIC, it is desired to have a high polarization on the spin-polarized protons. Therefore it is important to determine the spin polarization of the proton beam accurately. Conversely, if a visual observation of the particle beam can also be obtained in the same system it would benefit the beam diagnostic significantly. At the 12 o'clock location of RHIC a supersonic atomic hydrogen jet polarimeter is developed by the Collider Accelerator Division at the Brookhaven National Laboratory to measure the spin polarization of the proton beam. While the spin polarization being routinely measured, however, the passage of high-energy RHIC particle beams can also intersect and excite gas molecules to generate light by the process of luminescence through either atomic line emission or fluorescence. A preliminary attempt to obtain the proton beam profile based on this fluorescence from the hydrogen gas target was successful but with poor image quality on the RHIC proton beam [1]. Subsequence improvement in optics, CCD camera, and calibration resulted in a much improved image quality [2]. In this paper we describe the additional optimization on the optical system and the installation of a fiber-coupled spectrometer. The light collected can be spectrally resolved by a spectrometer, and more importantly it can be imaged by a CCD to produce a high quality spatial image of the proton beam as well as the $\mathrm{Au}$ ion beam to determine their sizes, position, movement, emittance, and other important parameters that are highly relevant to the RHIC accelerator. This novel yet truly noninvasive particle beam profile measurement technique promises to find applications not only at RHIC but also in most proton and ion beam accelerator facilities.

\section{RHIC PARAMETERS}

The RHIC at the Brookhaven National Laboratory collides heavy ion nuclei to create high density and temperature, and explore a regime of the possible deconfinment of quarks and gluons in the colliding nuclei. RHIC also collides intense beams of polarized protons, reaching transverse energies where the proton scatters as beams of polarized quarks and gluons. Two counterpropagating beams circulate the RHIC rings, designate as yellow and blue rings. The beam lifetime on both rings typically reaches several hours of operation followed by beam refilling for half hour or less. More on the physics goals of RHIC and the detail machine parameters can be found in reference [3]. In the last FY2008 runs (Run-8), RHIC collided polarized proton on polarized proton at 100 $\mathrm{GeV}$ particle energy (p-p run), deuteron at 101.9 $\mathrm{GeV} /$ nucleon on $\mathrm{Au}^{79+}$ at $100 \mathrm{GeV} /$ nucleon particle energy (d-Au run), and $\mathrm{Au}^{79+}$ on $\mathrm{Au}^{79+}$ at 2.5 and $4.6 \mathrm{GeV} /$ nucleon particle energy (Au-Au run). Typically the RHIC runs at a revolution frequency (rings/sec) of $78 \mathrm{kHz}$ with a nominal 110 bunches for protons and deuterons, and 56 bunches for $\mathrm{Au}$ ions. Each proton/deuteron bunch and $\mathrm{Au}$ ion bunch contains $10^{11}$ particles/bunch and $10^{9}$ ions/bunch, respectively. Table 1 gives some of the RHIC machine parameters that are relevant in this study.

Table 1 Some standard RHIC run-8 machine parameters

\begin{tabular}{|l|c|c|c|}
\hline mode & $\mathrm{p}-\mathrm{p}$ & $\mathrm{d}-\mathrm{Au}$ & $\mathrm{Au}-\mathrm{Au}$ \\
\hline energy per nucleon $(\mathrm{GeV})$ & 100 & $101.9 \mathrm{~d} / 100 \mathrm{Au}$ & $4.6 / 2.5$ \\
\hline \# of bunch/ring & 111 & 95 & 56 \\
\hline \# of particles/bunch & $1.5 \times 10^{11}$ & $10^{11} \mathrm{~d} / 10^{9} \mathrm{Au}$ & $4 \times 10^{8}$ \\
\hline \# of particles/ring & $1.7 \times 10^{13}$ & $9.5 \times 10^{12} \mathrm{~d} / 9.5 \times 10^{10} \mathrm{Au}$ & $2.2 \times 10^{10}$ \\
\hline$\beta^{*}$ betatron function at injection $(\mathrm{m})$ & 1.0 & 0.85 & 10.0 \\
\hline$\gamma$ at injection & 11.15 & 25.4 & 4.9 \\
\hline emittance $\left.(\pi-m m-m m-r a d)^{-1}\right)$ & $20-25$ & $17-30$ & $40-60$ \\
\hline $\mathrm{L}_{\text {peak }}\left(\mathrm{cm}^{-2}\right.$ sec $\left.^{-1}\right)$ & $3.5 \times 10^{31}$ & $25 \times 10^{28}$ & $3.5 \times 10^{23}$ \\
\hline revolution frequency (rings/sec) $^{23}$ & 78189 & 78189 & 78189 \\
\hline Bunch period $(\mathrm{ns})$ & 115 & 134 & 228 \\
\hline Bunch width $(\mathrm{ns})$ & 8.3 & $2.5 \mathrm{~d} / 10 \mathrm{Au}$ & $\sim 10$ \\
\hline Total \# of particles per second & $1.3 \times 10^{18}$ & $7.41 \times 10^{17} \mathrm{~d} / 7.41 \times 10^{15} \mathrm{Au}$ & $1.75 \times 10^{15}$ \\
\hline
\end{tabular}

\section{THE POLARIZED HYDROGEN GAS JET}

A state-of-the-art Hydrogen-jet (H-jet) polarimeter has been built and used for the absolute spin polarization measurements of the proton beams in RHIC [4]. It is in operation prior to the implementation of the optical imaging system that we are going to describe in the following sections. The proton target in the polarimeter is a free atomic hydrogen beam, which crosses the RHIC beam orthogonally in the vertical direction. The polarimeter consists of 9 identical vacuum chambers vertically stacked together and differentially pumped. A schematic of the polarized hydrogen gas jet target is shown in Figure 1(a). In the top dissociator stage atomic hydrogen is produced through an RF discharge at a frequency of $21 \mathrm{MHz}$ with an input power of 180 Watts. The gas is passed downward through a conical nozzle $2 \mathrm{~mm}$ in diameter cooled to a temperature of $70{ }^{\circ} \mathrm{K}$. Two RF transitions at $1.43 \mathrm{GHz}$ and $14 \mathrm{MHz}$ force an interchange in the hyperfine state populations and provide the positive and negative spin polarization respectively. The collimated atomic beam with electron spin $+1 / 2$ are selected as they pass through two pairs of sextupole magnets and focused at the center of the scattering chamber. Atoms with opposite spin state are defocused and blocked. Only the spin $+1 / 2$ hydrogen atoms 
are participated in the spin polarization measurement that is based on elastic proton-proton scattering in the CoulombNuclear Interference region [5]. The RHIC proton beam polarization is measured by analyzing the symmetry of the recoil trajectory on the polarized hydrogen target. Due to particle identity, the absolute polarization of the accelerated proton beam can be directly interpreted by the proton target polarization. The scattering chamber encompasses a holding field magnet at 0.12 Tesla with a uniformity of 1 part in $10^{3}$ over a $40 \mathrm{~mm}$ region. This was constructed with two bucking Helmholz coils providing a central field region yet cancelling the radial field in order to minimize the effect of the magnetic field on the trajectory of the scattered low energy protons. Below the scattering chamber is the Breit Rabi Polarimeter (BRP) that consists of additional pairs of sextupole magnets, RF transitions, a beam blocker followed by a beam intensity monitor. This polarized atomic hydrogen beam source produces a cylindrical column of $\mathrm{H}-$ jet target with a calculated aerial intensity of $(1.3 \pm 0.2) \times 10^{12}$ atoms $/ \mathrm{cm}^{3}$ thickness at a supersonic velocity of $1562 \mathrm{~m} / \mathrm{sec}$. The profile of the atomic beam measured by a compression tube technique [6] typically reaches a diameter of $6.5 \mathrm{~mm}$ FWHM. The H-jet has been operated with record intensity of $(12.4 \pm 1.0) \times 10^{16}$ atoms $/ \mathrm{sec}$. Proton beam polarization of $\mathrm{P}_{+}=0.957 \pm 0.001$ and $\mathrm{P}_{-}=0.959 \pm 0.001$ have been measured by the BRP, while the maximum theoretical polarization at 0.12 Tesla is 0.961 [7].

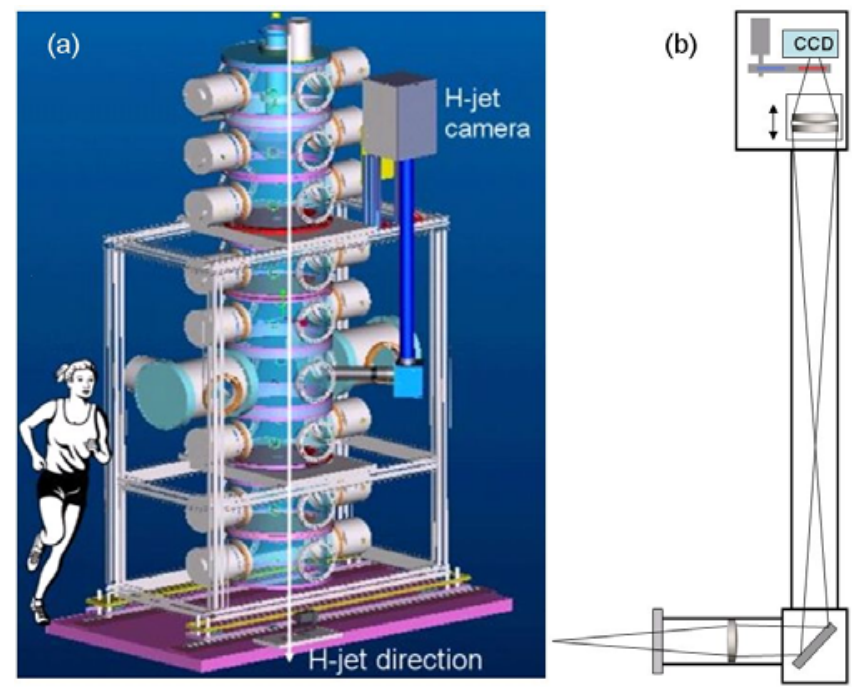

FIG. 1. (Color online) (a) H-jet with external modification. (b) Optical imaging system, not to scale.

The entire $\mathrm{H}$-jet column is mounted on a track capable of moving $\pm 5 \mathrm{~mm}$ orthogonal to the RHIC beam path for beam studies, see Figure 2. The H-jet vacuum in the beam chamber is $\sim 2 \times 10^{-8}$ Torr with the H-jet on and $\sim 4 \times 10^{-9}$ Torr with the $\mathrm{H}$-jet off. The former number guarantees adequate density for the reliable $\mathrm{H}$-jet operation for spin polarization measurement when proton beams are running at RHIC. While the latter number is important should the jet stay on the beam line when RHIC is running in heavy ions mode. It was known from early RHIC heavy ion beam runs that electron cloud developed in poor vacuum condition. Not only the vacuum was spoiled, the ion beam also deteriorated resulting in large background signals for most RHIC experiments [8]. However, even at a high vacuum condition of $10^{-9}$ Torr, the passage of the high-energy ion beam still interacts with the residual gas but with negligible effects on routine RHIC beam operation. Ironically, in the latter part of this study we make use of the ion beam induced fluorescence emitted by residual gas molecules to implement our ion beam profile monitor.
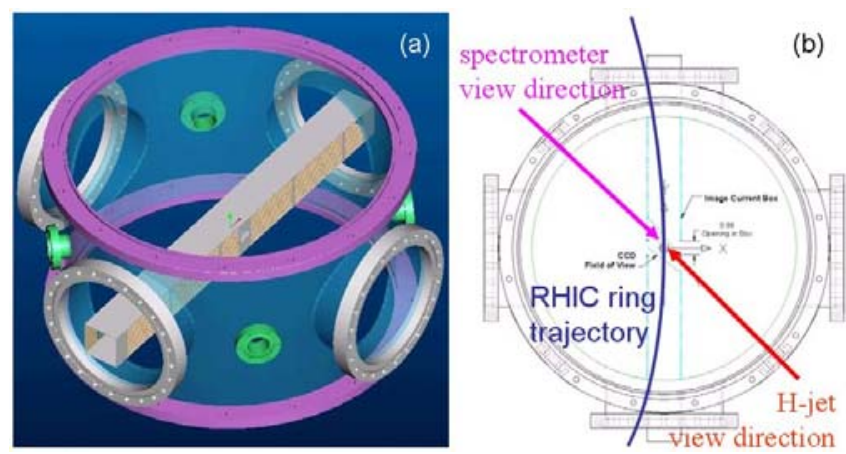

FIG. 2. (Color online) (a) Drawing of the H-jet interaction chamber, showing the rf wire cage at the beam center. (b) Cross-sectional view of the chamber showing the image light paths in relation to the RHIC beam trajectory.

\section{THE LUMINESCENCE MONITOR}

The H-jet polarimeter is located at the 12 o'clock of RHIC. Because of the small jet target thickness, it is operated continuously without any interference with other experiments or the RHIC beams. Conversely, the optical luminescence monitor attached to the $\mathrm{H}$-jet polarimeter is also operated continuously and independently without interfering the RHIC operation or the polarimeter operation. Although a version of luminescence beam profile monitor is in use in high-energy colliders $[9,10]$, it requires an injection of undesirable high pressure $\left(\sim 10^{-6}\right.$ Torr $)$ of gas in the accelerator. While beam profile is a primary goal of our project, measurements of the optical signals originated other than from hydrogen could lead to the possible detection of impurities such as oxygen or water molecules. This capability would allow us to examine the H-jet operation by observing the optical signals from impurities. Two independent light collection paths are defined at the $\mathrm{H}$-jet interaction chamber for $\mathrm{H}$-jet imaging and spectral identification, see Figure 2(b). Because no viewport is available for a 90-degree observation of the RHIC beam, both optical paths sustain a 45 degrees viewing angle to the beam center.

\section{A. HYDROGEN LINE SPECTRUM}

Excitation of the spin-polarized hydrogen by the high-energy proton beam produces the familiar hydrogen Balmer series lines emission at the visible wavelengths of $656.3 \mathrm{~nm}\left(\mathrm{H}_{\alpha}\right), 486.2 \mathrm{~nm}\left(\mathrm{H}_{\beta}\right), 434.1 \mathrm{~nm}\left(\mathrm{H}_{\gamma}\right)$, and shorter wavelengths associated with higher energy quantum states. As a secondary effect of this proton-proton interaction, the 
luminescence induced by the passage of the proton beam is used to determine the vertical dimension of the proton beam and subsequently to validate the emittance conservation in the accelerator. Prior to the H-jet spectral investigation, the emission spectrum of the hydrogen dissociator is measured by a fiber-coupled spectrometer through a viewport on top of the H-jet in the RHIC tunnel. The spectrometer has a spectral resolution of $0.8 \mathrm{~nm}$. It is found that a weak but broad spectral pedestal is riding on the entire visible spectrum limiting the detection of hydrogen Balmer series lines to below $430 \mathrm{~nm}$, see Figure 2(a). It is speculated that such broad spectral feature is the result of rf plasma excitation from the dissociator. Within the spectral resolution of the spectrometer, all measured spectral lines agree with published values. Should the $\mathrm{H}$-jet be contaminated with trace of other impurity gas, the spectrum of the H-jet dissociator is measured when small trace of oxygen is purposely introduced into the dissociator. Figure 3 (b). The well-known oxygen emission lines are clearly observed in the otherwise spectral pure hydrogen dissociator. Nonetheless, in normal operation the dissociator contains no impurity spectral lines other than the hydrogen Balmer series lines and the broad spectral pedestal.

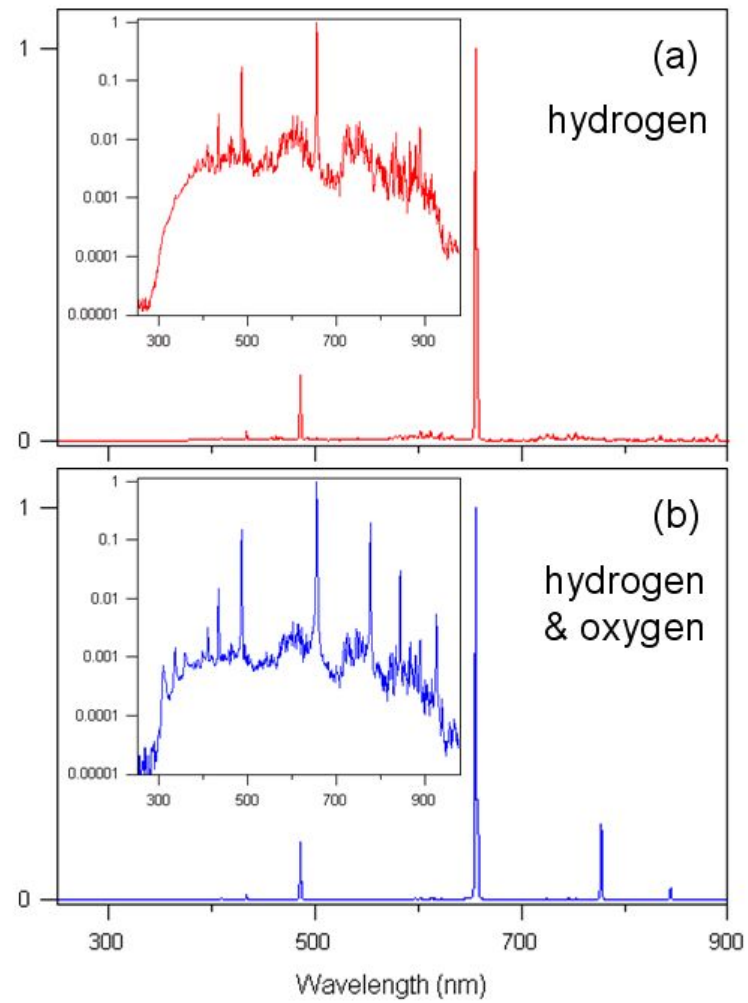

FIG. 3. Dissociator spectrum measured on top of the H-jet, inset shows the spectrum in logarithmic intensity scale. All normalized to the $656.3 \mathrm{~nm} \mathrm{H}_{\alpha}$ line, (a) normal operation, (b) with oxygen purposely introduced in the dissociator.

\section{B. IMAGING SYSTEM}

The optical system is a simple image relay arrangement. However, based on the physical geometry of the hydrogen jet and the available mounting locations, the resulting design ended up with a total optical path length of
$2.475 \mathrm{~m}$, an effective focal length of $0.1 \mathrm{~m}$ and an image demagnification of $\sim 0.25$, see Figure 1(b). To minimize damage to the CCD camera, a first-surface elliptical mirror was use to steer the optical beam path upward by $\sim 1.8 \mathrm{~m}$ from the proton beam center. The maximum Field of View (FOV) is limited by the $35 \mathrm{~mm}$ diameter of a fused silica viewport located 11-inch from the beam center. All three relay lens are AR-coated achromat $75-\mathrm{mm}$ in diameter with focal length of $300 \mathrm{~mm}$ and a thickness of $19.8 \mathrm{~mm}$ (Edmund P45-418), all mounted in a black anodized tube mount (ThorLabs SM3L10). The compromise on the light collection efficiency limited the depth of field to $\pm 4 \mathrm{~mm}$. The light emission solid angle defined by the optics is $1.4 \times 10^{-3}$. The transmittance of the entire optical system is shown in Figure 4. At first, the image size is estimated by knowing the dimension of the rf image-current cage and wires positioned at both side of the beam center inside the chamber, see also Figure 2. Based on calculations of the optical system and images obtained of the near and far wires, a theoretical center was determined and used for beam imaging studies. The entire light-tube and enclosure was constructed of black anodized aluminum to prevent stray light from entering the imaging system.

A Meade 416 XTE camera with a maximum exposure time of 2 hours is used to capture the images; it uses a Kodak KAF-0401E - 768 x 512 pixels CCD chip. The CCD has a quantum efficiency of 0.45 at $656 \mathrm{~nm}$ and the normalized spectral response of the CCD is measured and shown in Figure 4. The CCD has a 16-bit monochrome image depth -65536 brightness level. It is Peltier cooled to $-10^{\circ} \mathrm{C}$ to improve the signal-to-noise. It has a read nose of $<15$ electrons rms and a full well capacity of 85000 electrons, which corresponds to a calculated 1.3 electron charge per brightness level. Similarly, at the $\mathrm{H}_{\alpha}$ wavelength of $656 \mathrm{~nm}$ a calculated 2.88 photons/pixel/CCD-brightnesslevel is obtained. The Meade camera control unit and a local control desktop PC are mounted nearby the H-jet. Image data is sent via a SCSI interface to the PC running Windows XP. The Meade Pictor 7.25 software allows a user to control the camera data acquisition process, however, no automatic image collection sequence can be implemented. The PC is fully controlled using an Ethernet connection to the network running Windows XP remote desktop connection. A motorized order sorting filter wheel is placed in front of the CCD camera with various interference band-pass filters (ThorLabs), $656 \mathrm{~nm}, 600 \mathrm{~nm}, 486 \mathrm{~nm}, 460 \mathrm{~nm}$, and $390 \mathrm{~nm}$ for qualitative spectral selection. Except the $600 \mathrm{~nm}$ that has a $40 \mathrm{~nm}$ bandwidth, all filters have a $10 \mathrm{~nm}$ bandwidth. The last lens placed in front of the camera is mounted on a remotely controlled moveable stage for image focus adjustment.

\section{FIBER-COUPLED SPECTROMETER SYSTEM}

To quantify the $\mathrm{H}$-jet emission spectrum and to find the spectral signature of impurity gas, a FC fibercoupled collection lens (Micro Laser Systems, FC20-T) is installed directly across the image path of the H-jet camera but in front of a $35 \mathrm{~mm}$ opening fused silica viewport, see 
Figure 2(b). The fiber-coupled lens has a $23 \mathrm{~mm}$ aperture and is mounted on a 2 -axis motorized tilt holder to maximize the light coupling efficiency. The light collected is coupled into a light-tight 70 meters long $200 \mu \mathrm{m}$ diameter optical fiber (ThorLabs BFH22-200) to a non-radiation area outside the RHIC tunnel. This long fiber has a good visible to near UV transmission, see Figure 3. The high $\mathrm{OH}$ content of the fiber provides radiation resistance to better than 1 Mrad measured total gamma radiation dose with negligible transmission loss. The other end of the optical fiber is coupled to a fiber-coupled spectrometer (Ocean Optics QE65000, slit width $25 \mu \mathrm{m}$ ) with 5 minutes integration time. The $\mathrm{H}$-jet spectrum is collected and automatically saves onto a PC every five minutes.

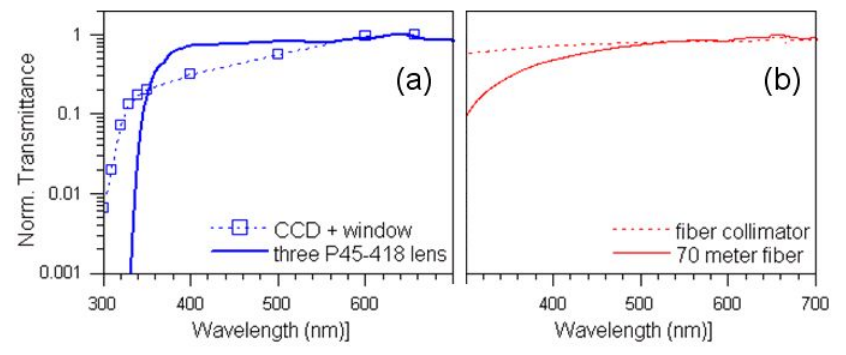

FIG. 4. (Color online) Normalized transmittance of (a) the H-jet imaging system, and (b) the fiber optics spectral collection path.

\section{CALIBRATION OF THE CCD CAMERA}

Since the optical imaging system is mounted to the external of the $\mathrm{H}$-jet as an attachment at a much later time and it is undesirable to break the vacuum for a direct image size calibration, therefore, a complete mockup is constructed in the laboratory to evaluate the optical FOV, the sensitivity of the camera, and their spectral responses. It is discovered that both CCDs, in the field and the mockup, have condensation formed on the windowless CCD chip when the unit is cooled to its operating temperature. The sensitivity of the camera is measured upon return for repair. The light output of a single mode fiber-pigtailed $650 \mathrm{~nm}$ LED is used to determine its photon sensitivity as well as the spectral transmission of the entire optical system. The collimated LED light output is attenuated by calibrated neutral density filters to a known number of photons measured by a Newport 1930C optical power meter. The light is then impinges on the CCD camera. With a measured spot size and the known number of photons, we determined the calibration of the camera to be $3.64 \times 10^{4}$ photons/CCDbrightness-level $/ \mathrm{mm}^{2}$ at $650 \mathrm{~nm}$. This experimental calibration corresponds to a 2.94 photons/pixel/CCDbrightness-level, which is in good agreement with the calculated value of 2.88 based on the specification of the CCD. A measured read noise of 8.8 brightness level is obtained at $-10^{\circ} \mathrm{C}$, which corresponds to 11.6 electrons rms noise, in reasonable comparison to the specification of $<15$ electrons rms. The overall optical transmission of the imaging path is 0.58 , and the camera's FOV is $26.9 \mathrm{~mm} \mathrm{x}$ $17.9 \mathrm{~mm}$. This FOV applies to all H-jet images presented in this study. An image-scaling factor of $34.9 \mu \mathrm{m} / \mathrm{pixel}$ is established. In addition, the proper orientation of the image was also determined.

\section{LUMINESCENCE WITH HYDROGEN-JET}

During the RHIC operation, the $\mathrm{H}$-jet luminescence camera monitors the RHIC beam noninvasively and measures its beam characteristics. These measurements include transverse beam size, beam emittance, beam position, and beam intensity. The $\mathrm{H}$-jet luminescence camera system can provide real-time guidance for RHIC beam tuning, injection, and operation. It serves not only to confirm the RHIC beam characteristics but also to provide a true image view of the RHIC beams.

\section{A. BEAM MONITORING}

When two cylindrical-like beam, proton beam and $\mathrm{H}$-jet, intersect at the center of the interaction chamber, the image of the dominant $\mathrm{H}_{\alpha}$ luminescence emission is filtered and collected by the CCD camera. A time lapsed image sequence on the order of 20 minutes are taken showing the motion of the two proton beams with respect to each other during injection and ramping, see Figure 5, where yellow and blue beams contain $65 \times 10^{11}$ and $85 \times 10^{11}$ polarized protons per bunch in 108 bunches, respectively.

Alternately, when the RHIC beams are stationary, the H-jet column can be rolled on its track to independently select either the blue or the yellow beam for spin polarization measurement. Conversely, the luminescence of the RHIC beams are also being selected, shown in Figure 6; where the hydrogen supersonic jet is propagating from top to bottom in these and all other $\mathrm{H}$-jet images. These qualitative image sequences clearly illustrated one can use the secondary effect of the $\mathrm{H}$-jet, hydrogen luminescence, for routine beam monitoring.
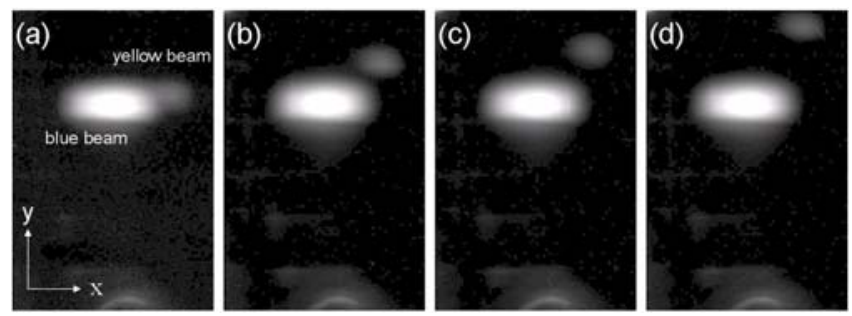

FIG. 5. Sequences of H-jet images during RHIC beam injection and ramping. The blue proton beam is being moved away from the yellow beam. The exposure of the camera is set to 60 seconds and all mages are in logarithmic intensity scale to highlight the blue beam in the background. The field of view is $26.9 \mathrm{~mm} \times 17.9 \mathrm{~mm}$.
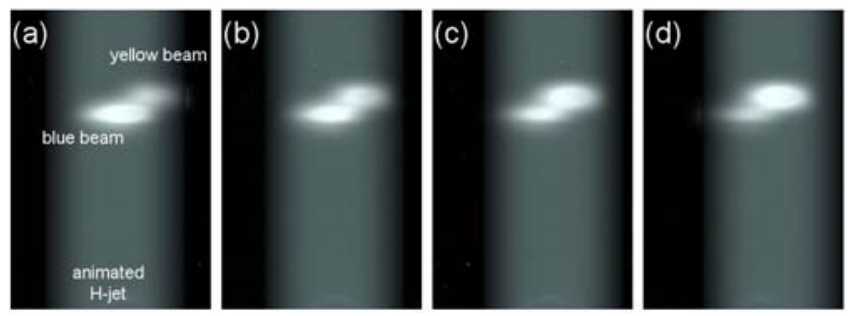

FIG. 6. (Color online) Sequences of H-jet images when the entire H-jet column is displaced away from the RHIC beam center by $2.5 \mathrm{~mm}$ to the 
right, (a)-(d). An animated $\mathrm{H}$-jet column is overlaid on each image to illustrate the translation effect, the hydrogen jet is propagating from top to bottom and the proton beams are counter propagating from left to right, respectively. The exposure of the camera is set to 30 seconds. All mages are displayed in linear intensity scale. The $\mathrm{H}_{\alpha}$ line is selected using a 656 nm interference filter.

\section{B. RHIC PROTON BEAM PROFILE}

The $\mathrm{H}$-jet luminescence image gives a qualitative visual observation of the RHIC beam. Moreover, using the calculated FOV and photon sensitivity of the CCD, the processed image provides quantitative information relevant to the circulating beam. Figure 7 shows a $656 \mathrm{~nm}$ filtered luminescence profile on a typical polarized proton beam. Because the proton beam bunch width $(\sim 8 \mathrm{~ns})$ and bunch period $(\sim 110 \mathrm{~ns})$ are both much greater than the $\sim 10 \mathrm{~mm}$ hydrogen jet diameter, only information in the y-axis column is relevant to the vertical size of the circulating $\mathrm{RHIC}$ beam. Information obtained in the $\mathrm{x}$-axis corresponds to the width of the supersonic $\mathrm{H}$-jet column instead. This width measured $4.52 \mathrm{~mm}$ FWHM on the raw data, which corresponds to $6.57 \mathrm{~mm}$ FWHM in diameter $(2.8 \mathrm{~mm} \sigma)$ after corrected for the 45-degree viewing angle. However, we should caution that when the proton beam traverses on the outer edge of the cylindrical $\mathrm{H}$-jet, the apparent width of the H-jet image decreases. This optical measurement technique is in good agreement with two independent but complex measurement techniques where first the compression tube $\mathrm{H}$-jet column itself is moved to see the relative position of the blue beam and yellow beam [11], and second the entire $\mathrm{H}$-jet-target system is moved while the event counts detected by the recoil spectrometer versus position is recorded. All three independent measurements agree well. The target profile measurement using RHICbeam is important to find the best collision point and estimate the fraction of the unpolarized proton background.

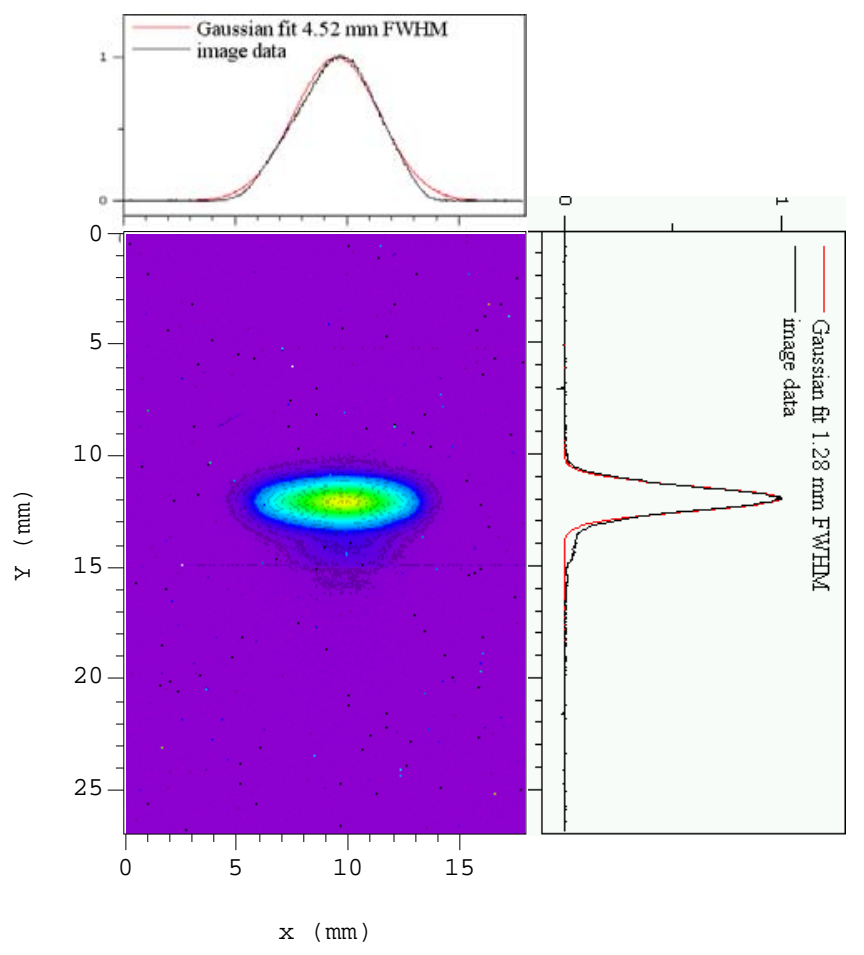

FIG. 7. (Color online) False color image and the Gaussian fit of a raw H-jet luminescence profile for RHIC blue ring, $\mathrm{H}_{\alpha} 656 \mathrm{~nm}$ filtered, RHIC fill \#9992, 111 bunches/ring, 30 second exposure. A total of $3.1 \times 10^{19}$ protons induced the luminescence of $2.2 \times 10^{8}$ photons collected on the CCD.

In contrast, the y-axis projection of the beam profile as produced by the luminescence monitor gives a vertical proton beam size of $1.28 \mathrm{~mm}$ FWHM $(0.54 \mathrm{~mm} \sigma)$, which is in good agreement with the value expected at this location. The observed vertical proton beam profile has a near Gaussian shape. However, careful examination on all raw image data show a tail developed on the flow direction of the $\mathrm{H}$-jet, see the color image of Figure 7. In addition, the thin wires of the rf cage in the foreground sometime accidentally but slightly obscure the raw images. This image distortion is apparent in Figure 8 where hum and dip are observed at the $\mathrm{x}=15$ to $16 \mathrm{~mm}$ location. Despite the H-jet image occasionally interfered by the rf wires, the $\mathrm{H}$-jet image remains clear and informative.

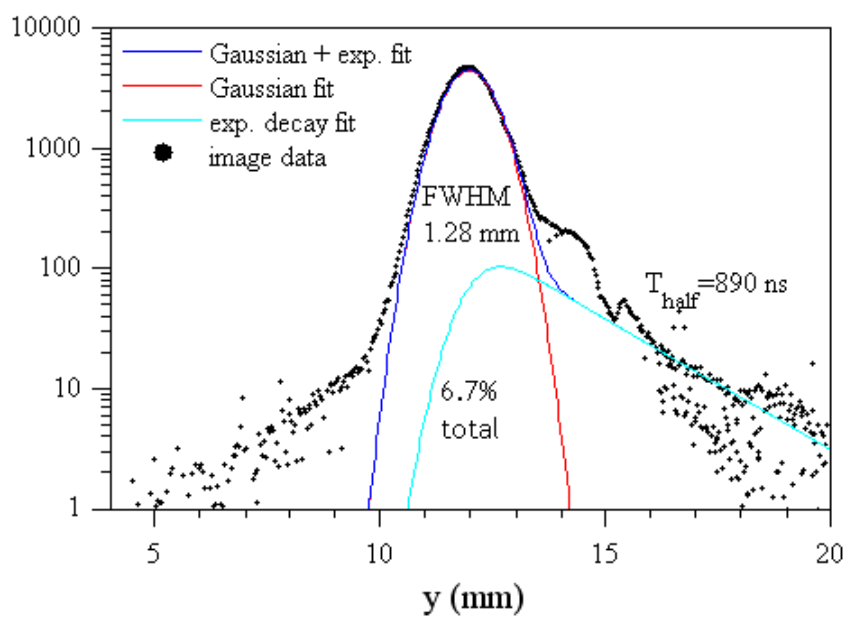


FIG. 8. (Color online) Detail on the y-axis image data shown in Figure 7 where a Gaussian of $1.28 \mathrm{~mm} \mathrm{FWHM}\left(\sigma_{\mathrm{y}}=0.54 \mathrm{~mm}\right)$ is fitted to the main peak (red line), an exponential decay of $\mathrm{T}_{1 / 2}=0.89 \mu$ s to the tail (cyan line), and the overall fit of the data - convolution of Gaussian fit and the exponential decay (blue line). Note that the vertical axis is in logarithmic scale.

Based on the hydrogen gas jet's supersonic velocity of $1562 \mathrm{~m} / \mathrm{s}$ and the dimension on the field of view, a $0.89 \mu \mathrm{s}$ exponential decay rate was obtained after devolution. It is speculate that this corresponds to the lifetime of an excited state of the hydrogen atom. Nevertheless, the amount of this excited state (cyan line of Figure 8) contributes only $\sim 7 \%$ of the total integrated intensity output of a typical $\mathrm{H}$-jet luminescence profile. Although the measured lifetime drops slightly to $\sim 0.79 \mu \mathrm{s}$ when the image of the $\mathrm{H}_{\beta} 488 \mathrm{~nm}$ is filtered, the exponential tail remains clearly noticeable in logarithmic intensity scale.

\section{COMPARISON WITH IPM}

Independent verification of the proton beam width is achieved by comparing the $\mathrm{H}$-jet image measurement with the Ionization beam Profile Monitor (IPM) at the 2 o'clock location at RHIC. Figure 9 plots the raw blue ring IPM data of RHIC fill \#9992 - image data of Figure 7. A Gaussian curve fit to the data gives a beam width of $\sigma_{\text {IPM }}=1.26 \mathrm{~mm}$. The calculated betatron function $\beta_{\text {IPM }}$ at the IPM location is 62.6 meters, therefore the normalized $95 \%$ transverse emittance $\varepsilon_{I P M}$ can be calculated from

$$
\varepsilon_{I P M}=\frac{6 \pi \gamma \sigma_{I P M}^{2}}{\beta_{I P M}},
$$

where the relativistic factor $\gamma$ equals 106.8. This formula gives a measured emittance of $16.2 \pi \cdot \mathrm{mm} \cdot \mathrm{mrad}$ from the IPM data. Alternatively, using a calculated betatron function at the $\mathrm{H}$-jet location of $\beta_{\mathrm{H}-\mathrm{jet}}=10.31$ meters and the proton beam size $\sigma_{\mathrm{H}-\mathrm{JET}}=0.54 \mathrm{~mm}$ measured by the $\mathrm{H}$-jet, the transverse emittance is calculated to be $18.0 \pi \cdot \mathrm{mm} \cdot \mathrm{mrad}$. This particular set of emittance approaches the RHIC design emittance of $10 \pi \cdot \mathrm{mm} \cdot \mathrm{mrad}$. Nevertheless, the agreement between the H-jet and the IPM measurements is good. It is standard to use the emittance conservation rule to infer the RHIC beam size and other parameters in the ring. The $\mathrm{H}$-jet images provide a cross check with other instruments and more importantly to validate the assumption of emittance conservation in the RHIC ring.

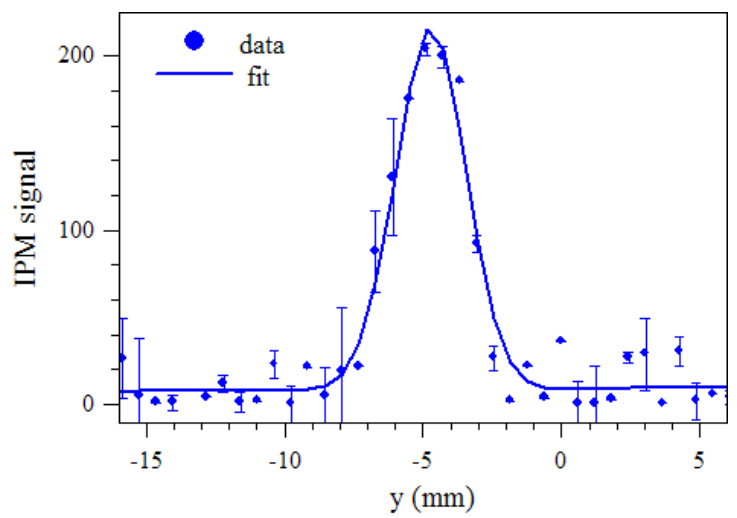

FIG. 9. (Color online) RHIC blue ring averaged vertical IPM data for fill \#9992.A Gaussian curve fit to the data gives a beam width of $2.97 \mathrm{~mm}$ FWHM (1.26 $\mathrm{mm} \sigma)$, some representative error bars of the raw data are shown.

To shed more light on the feature and the capability of the of the H-jet luminescence monitor, Figure 10 plotted the profile when both the yellow and blue circulating beams are vertically separated by $\sim 5 \sigma$ apart, which is a typical minimum separation before the onset of beam-beam coupling. Once again Figure 11 plotted the IPM results of these 2 beams and Table 2 summarized the results of the comparison. We note that beam sizes obtained from IPM and $\mathrm{H}$-jet in two different locations at the RHIC ring are inherently different because of beam optics and beam propagation. However the transverse emittance is a constant value depends only on the relativistic $\gamma$ factor and the betatron function. But the transverse emittance depends sensitively on the square of the measured beam spot size, shown in equation (1). Thus the emittance can vary significantly on various RHIC beam runs. It is interesting to note that measurement made by $\mathrm{H}$-jet indicates the blue beam size is slightly $(\sim 10 \%)$ larger than yellow; however the IPM results suggests otherwise. In addition, the measurement on the beam separation is also slightly different; H-jet gives a $5.2 \sigma$ between the blue and yellow beams, while the IPM infers a $4.4 \sigma$. These differences remain to be reconciled. 


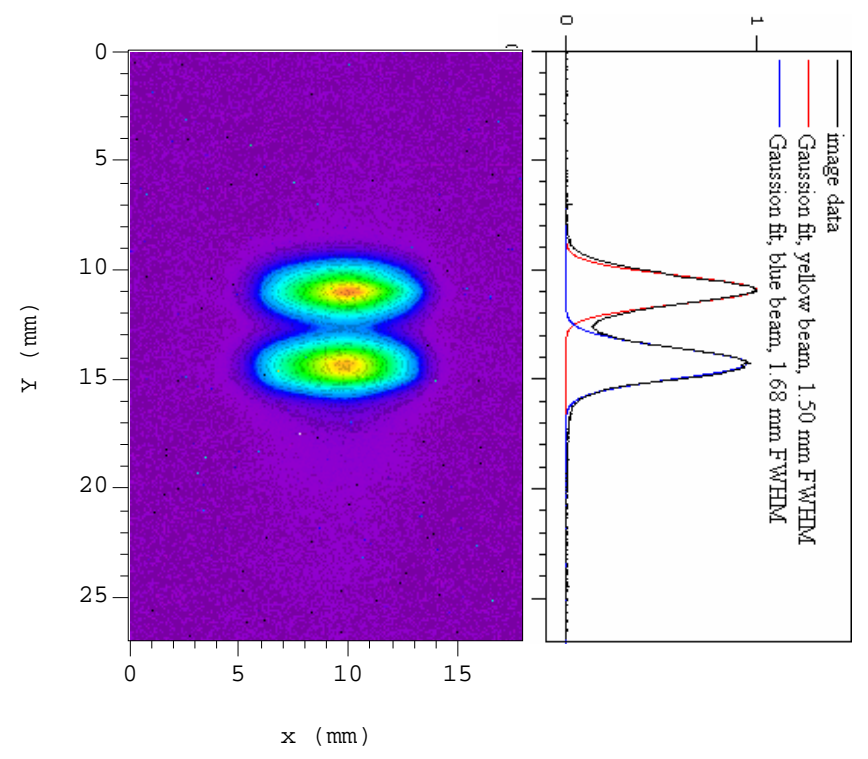

FIG. 10. (Color online) False color image and the Gaussian curve fit of a raw $\mathrm{H}$-jet luminescence profile for both RHIC rings. Top (bottom) is yellow (blue) beam. $\mathrm{H}_{\alpha} 656 \mathrm{~nm}$ filtered, RHIC beam fill \#10000, 111 bunches/ring, 30 -second exposure, total of $4 \times 10^{19}$ protons.

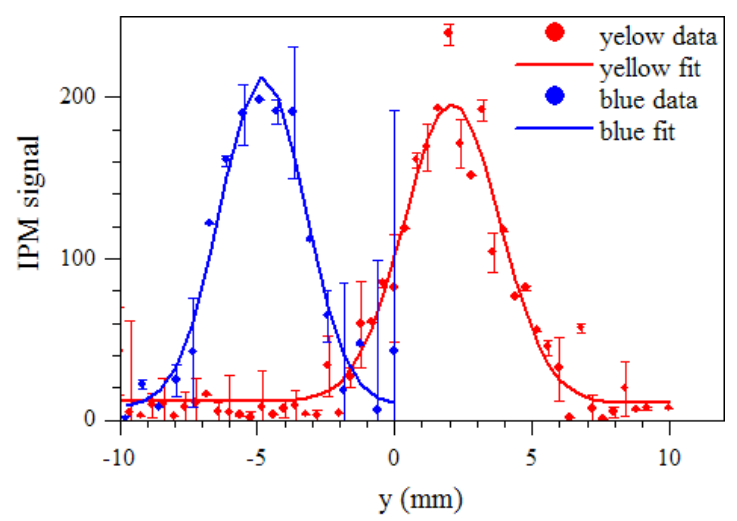

FIG. 11. (Color online) RHIC ring averaged vertical IPM data for fill $\# 10000$. A Gaussian curve fit to the data gives a blue beam width of 3.59 $\mathrm{mm}$ FWHM (1.53 $\mathrm{mm} \sigma)$ and yellow beam width of $4.04 \mathrm{~mm}$ FWHM (1.72 $\mathrm{mm} \sigma$ ), some representative error bars of the raw data are shown.

Table 2 Comparison between H-jet and IPM beam profiles for p-p run

\begin{tabular}{|l|l|l|l|l|l|l|}
\hline RHIC p-p runs & \multicolumn{2}{|c|}{ fill \# 9992} & \multicolumn{3}{c|}{ fill \# 10000 } \\
\hline beam profiler & \multicolumn{1}{|c|}{ H-jet } & \multicolumn{1}{c|}{ IPM } & \multicolumn{3}{c|}{ H-jet } & \multicolumn{2}{c|}{ IPM } \\
\hline RHIC beam & yellow & yellow & yellow & blue & yellow & blue \\
\hline$\sigma(\mathrm{mm})$ & 0.54 & 1.26 & 0.64 & 0.71 & 1.72 & 1.53 \\
\hline$\beta$ betatron function & 10.3 & 62.6 & 9.87 & 10.3 & 63.6 & 62.7 \\
\hline$\gamma$ function & 106.8 & 106.8 & 106.8 & 106.8 & 106.8 & 106.8 \\
\hline $\boldsymbol{\varepsilon}(\boldsymbol{\pi} \mathbf{~ m m ~ m r a d ) ~}$ & $\mathbf{1 8 . 0}$ & $\mathbf{1 6 . 2}$ & $\mathbf{2 6 . 6}$ & $\mathbf{3 1 . 4}$ & $\mathbf{2 9 . 9}$ & $\mathbf{2 3 . 7}$ \\
\hline center $(\mathrm{mm})$ & 12.0 & -4.7 & 10.9 & 14.4 & 2.1 & -4.8 \\
\hline beam separation & - & - & \multicolumn{3}{c|}{$5.2 \sigma$} & \multicolumn{2}{c|}{$4.4 \sigma$} \\
\hline
\end{tabular}

\section{BEAM INTENSITY DEPENDENCE}

Using the calibrated number of photons per pixel per brightness level at the center wavelength of $656 \mathrm{~nm}$, one can obtain the total photon production and the luminescence cross section which is estimated to be $\sim 10^{-21} \mathrm{~cm}^{2}$ for 100 $\mathrm{GeV}$ protons incident on atomic hydrogen. Albeit this small visible photon production cross-section, the emission process is linearly dependence on the proton intensity, see Figure 12, indicating the luminescence is a single step process.

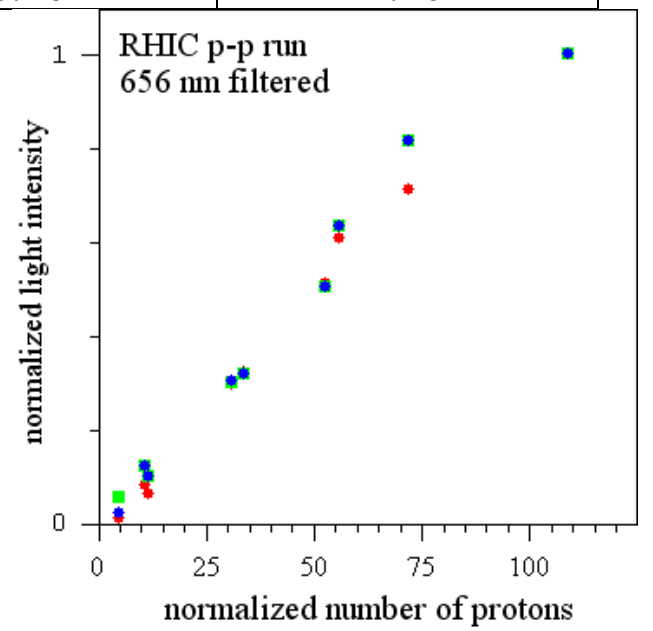

FIG. 12. Linearity of the RHIC blue ring intensity with the H-jet image integrated intensity after $656 \mathrm{~nm}$ filtered - accumulation of several run- 8 data.

To study the possibility of single bunch image detection by the $\mathrm{H}$-jet luminescence monitor, the sensitivity of the CCD is examined. The H-jet beam profile shown in Figure 7 (fill \#9992) corresponds to the luminescence from a total of $3.1 \times 10^{19}$ protons of $100 \mathrm{GeV}$ incidents on the 
atomic hydrogen gas jet. While a total of $2.2 \times 10^{8}$ photons are collected on the CCD. After considering the optical throughput of the imaging system $2.7 \times 10^{11}$ photons are emitting at the source point in a $4 \pi$ emission solid angle. Therefore the photon production efficiency is $8.6 \times 10^{-9}$ photon/proton (656 $\mathrm{nm}$ photons). A signal-to-noise ratio of 583 is established on this luminescence profile shown in Figure 7. A minimum light level required is then $3.8 \times 10^{5}$ photons per image. Based on this photon production efficiency, a single proton bunch can produce only $\sim 1$ photon while a single ring of protons produces $\sim 100$ photons reaching the CCD. However a single ring of protons could produces $\sim 10^{5}$ photons at the source point at the center of the interaction chamber. Therefore, using a MCP with image intensifier to increase the photon detection sensitivity and better light collection optics, single ring and single bunch of proton image can be obtained. However, single proton image are currently not feasible.

To further examine other possibility on the H-jet luminescence monitor, a detailed longitudinal bunch profile of the RHIC proton beam is extracted from a wall current monitor. Figure 13(a) plotted a subsection of the bunch structure for fill \#9992 (H-jet image shown in Figure 7(a)). Other than a fixed time offset and different bunch intensities, the blue and yellow beams have identical bunch spacing of $107.7 \mathrm{~ns}$ and a near Gaussian bunch width of 8.3 ns FWHM. Because a single proton bunch produces $\sim 570$ photons per pulse at the source point in $4 \pi$ direction and the emission is prompt relative to the luminescence lifetime or the bunch width, the peak optical power generated at the source point is thus $\sim 21$ nanoWatt per proton bunch. A typical photomultiplier tube can produce the single longitudinal bunch structure of the proton beam from the light collected at the $\mathrm{H}$-jet luminescence monitor.

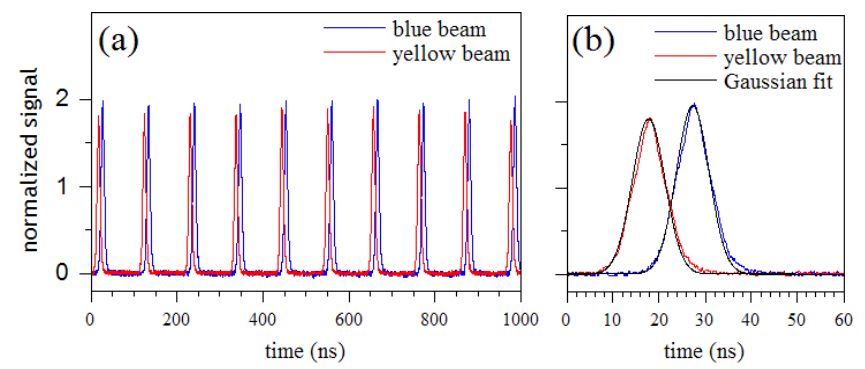

FIG. 13. (Color online) Longitudinal proton bunch profile of fill \# 9992 recorded by the wall current monitor; (a) a sub-section of the 111 bunch structure, showing the $107.7 \mathrm{~ns}$ bunch spacing, and (b) detail of the bunch shape, 8.3 ns FWHM both for the yellow and blue beams. Each bunch contains $\sim 1.2 \times 10^{11}$ protons of $100 \mathrm{GeV}$ per nucleon.

\section{E. H-JET SPECTRUM AND IMPURITY}

It is anticipated that the spectral contents from the hydrogen jet may include the ratio of atomic hydrogen to molecular hydrogen, oxygen, and water impurities. And it is hoped that knowing these impurity information one can provide an online tuning instrument for the $\mathrm{H}$-jet operation.

When RHIC is running at 111 bunches/ring during p-p run-8, the fiber-optics spectrometer installed directly across the image path of the H-jet camera successfully picked up this spectral information. The spectrometer is cooled to $-15{ }^{\circ} \mathrm{C}$ and integrated for 300 seconds after dark subtracted. A total of $\sim 3 \times 10^{20}$ protons at $100 \mathrm{GeV}$ energy is incident on the H-jet. Figure 14 shows a representative (RHIC fill \# 9992) but distinctive H-jet luminescence spectrum. In addition to the much stronger $656 \mathrm{~nm}$ hydrogen Balmer line, a total of 6 Balmer series lines down to $390 \mathrm{~nm}$ are observed. Within the spectral resolution of the spectrometer, all spectral lines and their relative intensities agree with literature values, see table inserted in Figure 14. A typical spectrum of the dissociator is also plotted for comparison. Both spectra are normalized to the $656 . \mathrm{nm} \mathrm{H}_{\alpha}$ emission line. Despite the spectrometer capable of resolving the hydrogen Balmer series down to the weak $\mathrm{H}_{\zeta}$ line, no other spectral impurity line is observed. In fact, the spectral purity of this hydrogen spectrum obtained from the relativistic proton induced luminescence is better than the dissociator source spectrum shown in Figure 2(a) and the black curve in Figure 14; no broad spectral pedestal is observed. Therefore the H-jet contains no measurable impurity gas.

In parallel to the $\mathrm{H}$-jet spectrum being collected on the fiber-optic path, the $\mathrm{H}$-jet camera also independently collects the luminescence profile. Because the light collection efficiency is limited by the entrance slit of the spectrometer and the size of the fiber-optical collimation lens, the $\mathrm{H}$-jet camera is slightly more sensitive (by a factor of $\sim 7$ ) than the spectrometer. Therefore, the H-jet image is spectrally selected by five interference filters to further examine the present of impurity gas. Identical exposure time of 5 minutes is set on both the $\mathrm{H}$-jet camera and the spectrometer. At the bottom of Figure 14 shows the unambiguous images of $656 \mathrm{~nm}, 486 \mathrm{~nm}$, and $390 \mathrm{~nm}$ for the specific hydrogen Balmer emission lines. All have a weak exponential decay tail following the flow direction of the H-jet. However, no clear H-jet images are seen on the other two spectral bands, $600 \mathrm{~nm}$ and $460 \mathrm{~nm}$ that are targeted to examine the broad but weak pedestal structure seen on the dissociator spectrum. Although this pedestal band is originally believed to have the signature of molecular hydrogen, our results suggested it is plasma in origin instead. The absent of a characteristic $\mathrm{H}$-jet image other than the hydrogen Balmer series lines leads us to establish an absolute upper limit of $0.01 \%$ on the ratio of the impurity gas relative to the hydrogen gas assuming the proton induced luminescence cross section of the impurity gas is comparable to that of hydrogen. 


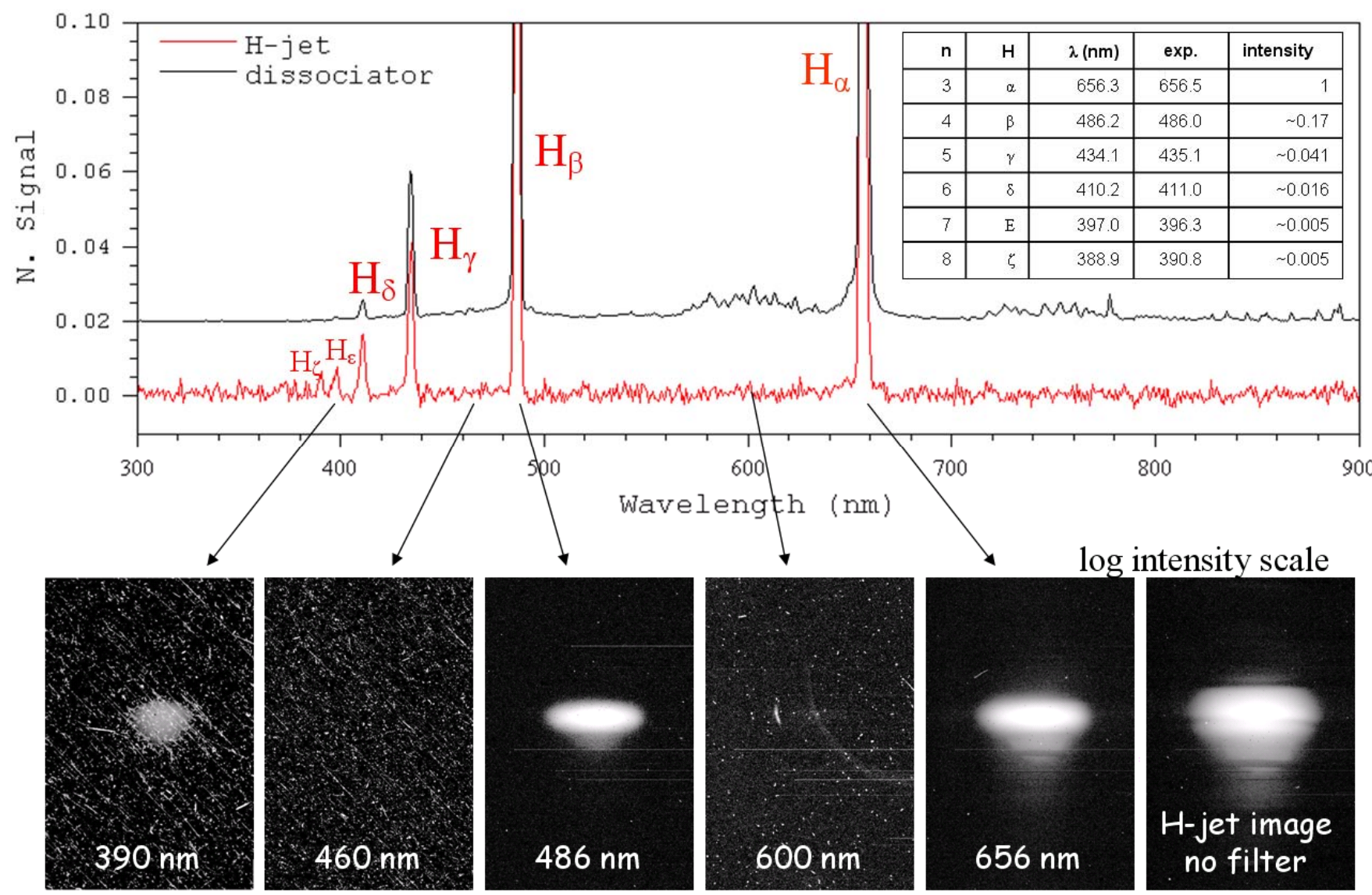

FIG. 14. (Color online) H-jet luminescence spectrum and image profiles for RHIC blue ring, RHIC fill \#9992, 111 bunches/ring. The spectrometer is cooled to $-15{ }^{\circ} \mathrm{C}$ and integrated for 300 seconds after dark subtracted. A typical spectrum of the dissociator is also shown for comparison; both spectra are normalized to the $656 \mathrm{~nm} \mathrm{H}_{\alpha}$ emission line. The dissociator spectrum is then vertically offset by 0.02 for clarity. A table compares the experimental $\mathrm{H}_{\mathrm{n}}$ lines to the literatures. The $\mathrm{CCD}$ of the $\mathrm{H}$-jet camera is also cooled to $-10^{\circ} \mathrm{C}$. A total of $\sim 3 \times 10^{20}$ protons at $100 \mathrm{GeV}$ is incident on the $\mathrm{H}$-jet.

\section{LUMINESCENCE WITHOUT HYDROGEN-JET}

During the $100 \mathrm{GeV}$ deuteron-gold (d-Au) runs and 4.6 GeV Au-Au runs of RHIC run-8, the hydrogen gas jet is intentionally turned off because no proton spin polarization measurement is anticipated. Thus the vacuum at the center of the interaction chamber reaches $10^{-9}$ Torr range. Yet on every d-Au and $\mathrm{Au}-\mathrm{Au}$ runs the $\mathrm{H}$-jet camera fortuitously recorded a streak of light image after a long time exposure. This light streak is absent, or below the sensitivity of the CCD camera, when only deuteron beam is circulating in the RHIC ring. We also note that this streak of light emission is absent when polarized proton beams are running with the $\mathrm{H}$ jet stayed off. Since the Au ions at RHIC are fully stripped $\mathrm{Au}$ ions, no fluorescence from the $\mathrm{Au}$ ion itself is expected. Therefore, these images are a clear signature of beam excitation and luminescence by the residual gas. Gold ion beam interacts with residual gas at RHIC has been examined in the early days of RHIC [8]; it was known that this beam gas interaction lowered the beam lifetime in RHIC. In recent years, RHIC runs in a high vacuum level of $10^{-9}$ Torr, much improved beam lifetime is obtained. Nevertheless, the highenergy ion beam still interacts with the residual gas but has negligible effects on routine RHIC beam operation. This beam-induced luminescence by residual gas represents a substantial step towards the realization of a simple yet truly noninvasive beam monitor of high-energy particle beam with unprecedented characteristics.

\section{A. BEAM PROFILE OF $100 \mathrm{GeV}$ GOLD ION}

Figure 15 shows the luminescence image of a typical $100 \mathrm{GeV} \mathrm{Au}$ ion beam (RHIC beam fill \#9642) passing through the center of the interaction chamber. No interference band pass filter is used; hence the CCD camera collected all luminescence light limited only by the spectral response of the CCD chip. At times when only deuteron is filling the ring, no light emission is detected. Therefore the luminescence is unambiguously due only to the passage of $\mathrm{Au}$ ion beams. In addition, the luminescence intensity depends linearly on the Au ion intensities, see Figure 16.

Five minutes camera exposure time is used to capture the image shown in Figure 15, which corresponds to the passage of a total of $1.4 \times 10^{18} \mathrm{Au}$ ions. Using the CCD brightness level calibration described in Section IV.D, a calculated total of $4 \times 10^{7}$ photons are collected on the CCD. After considering the optical throughput of the imaging system $3.87 \times 10^{10}$ photons are emitting at the source point in a $4 \pi$ emission solid angle. Therefore the photon production efficiency is $2.7 \times 10^{-8}$ photon/Au ion. Despite the gas density of the residual gas is significantly lower than that of the supersonic hydrogen gas jet, the photon production of $\mathrm{Au}$ 
ion is slightly higher (by a factor of 3 ) than that of the H-jet. It is speculated that it's the higher $Z$ (atomic number) of the $\mathrm{Au}$ ion leads to a larger luminescence cross-section.

This Au ion beam induced luminescence is a weak process, not only background image subtraction is needed some post imaging processing is also required to highlight the Au ion beam path. Image-J (NIH) processing software is used clean up the speckle pixels caused by ionization radiation induced transient on the CCD. The $y$-axis projection of the profile shown in Figure 15 gives a vertical $\mathrm{Au}$ ion beam size of $1.20 \mathrm{~mm}$ FWHM $(0.51 \mathrm{~mm} \sigma)$. Unlike the proton beam shape shown in Figures $7 \& 8$ where a decaying tail developed on the flow direction of the H-jet, the vertical Au ion beam shape has a near Gaussian beam shape with symmetric wings indicating no preferred residual gas flow direction. This wing-like broadening profile may also be an indication of space charge effects on the $\mathrm{Au}$ ion beam.

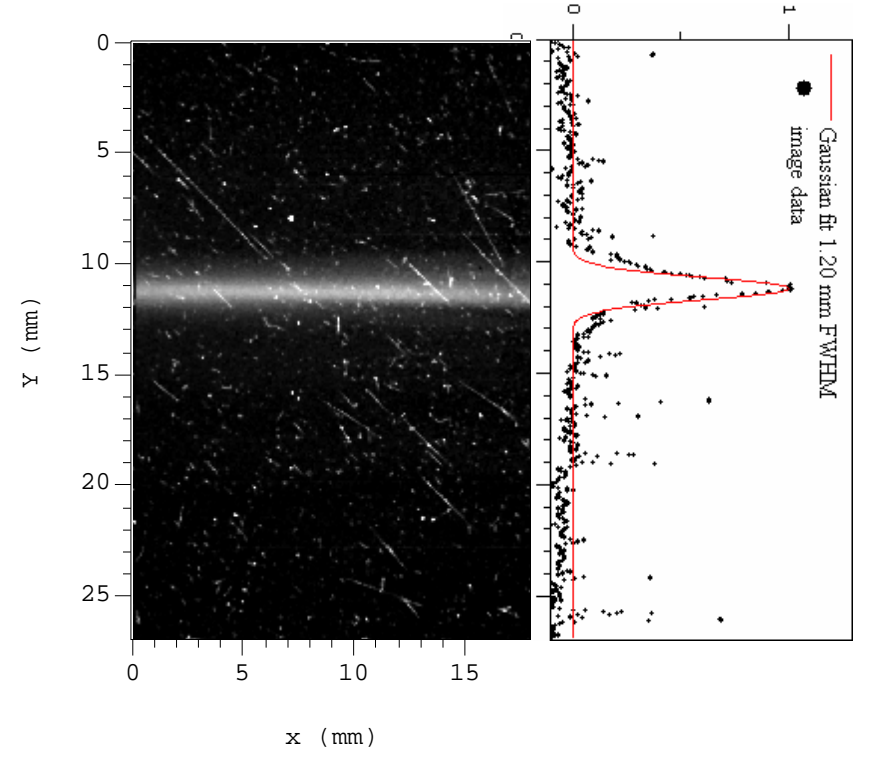

FIG. 15. (Color online) Camera image and the Gaussian fit of a luminescence profile for RHIC yellow ring, Au ion, during d-Au runs. No interference band-pass filter is used. RHIC fill \#9642, 95 bunches/ring, and 5 minutes exposure. A total of $60 \times 10^{9} \mathrm{Au}$ ion/ring induced the luminescence of $4 \times 10^{7}$ photons collected on the CCD.

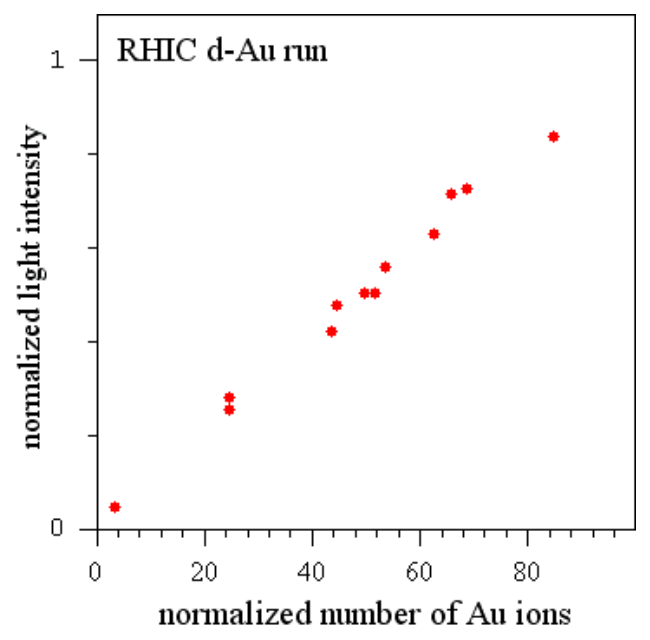

FIG. 16. Linearity of the RHIC yellow ring (Au ion) intensity with the Hjet image integrated intensity, no band-pass filters applied - accumulation of several run- 8 data.

Using the $\mathrm{Au}$ ion beam size an emittance of 55.8 $\pi \cdot \mathrm{mm} \cdot \mathrm{mrad}$ is calculated. However, an emittance of 13.5 $\pi \cdot \mathrm{mm} \cdot \mathrm{mrad}$ is obtained using the IPM data shown in Figure 17. Unlike polarized proton beam runs, on this particular $\mathrm{Au}$ ion beam run both the IPM and the luminescence monitor give a larger discrepancy comparing the average beam emittance of 17-30 $\pi \cdot \mathrm{mm} \cdot \mathrm{mrad}$ achieved during Run-8 dAu runs. Since a long exposure time is used to capture the $\mathrm{Au}$ ion beam images, it is probable that $\mathrm{Au}$ ion beam could have shifted with anticipated or unanticipated tuning of the RHIC accelerator. Consequently, the beam size derived from the luminescence profile is an absolute upper limit of the Au ion beam. Thus we established that the Au ion beam emittance must be less than $55.8 \pi \cdot \mathrm{mm} \cdot \mathrm{mrad}$. Table 3 summarized the comparison between the IPM and the luminescence monitor.

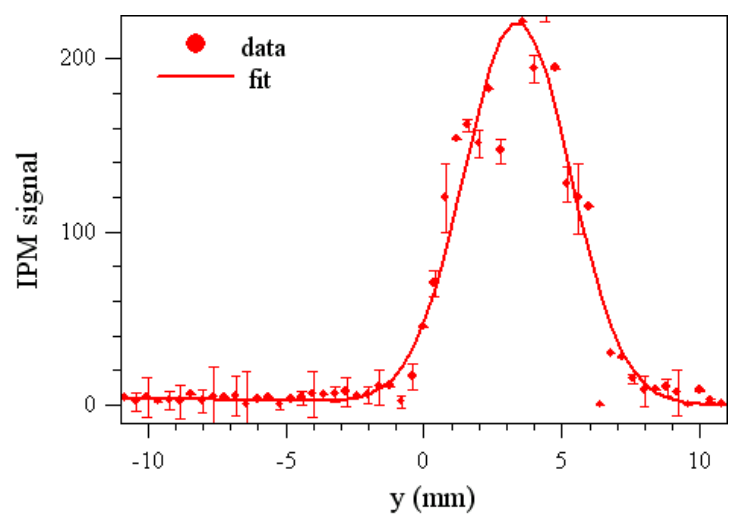

FIG. 17. (Color online) RHIC yellow ring, Au ion, averaged vertical IPM data for fill \#9642. A Gaussian curve fit to the data gives a beam width of $4.5 \mathrm{~mm}$ FWHM (1.9 mm $\sigma$ ); some representative error bars of the raw data are shown.

A longitudinal bunch profile of the RHIC d-Au run fill \#9642 is extracted from the wall current monitor. Figure 18(a) plotted a subsection of the bunch structure revealing a bunch spacing of $106.7 \mathrm{~ns}$. Although a total of 95 bunches 
are counted in one full ring, there are periodically missing bunches related to the RHIC accelerator. These bunch gaps are sometime intentionally constructed for specific RHIC experiments. Expanded view on the time structure of a single bunch, shown in Figure 18(b), unveils a 2.3 ns FWHM bunch width for the deuteron beam. However a single $\mathrm{Au}$ ion bunch contains sub-bunch structures and its overall bunch envelope are $\sim 10$ ns FWHM. Because a single $\mathrm{Au}$ ion bunch produces $\sim 17.5$ photons per pulse at the source point in $4 \pi$ direction, the light intensity generated at the source point is thus $\sim 0.5$ nanoWatt peak optical power per $\mathrm{Au}$ ion bunch. A high gain photomultiplier tube can potentially produce the single longitudinal bunch structure of the Au ion beam.

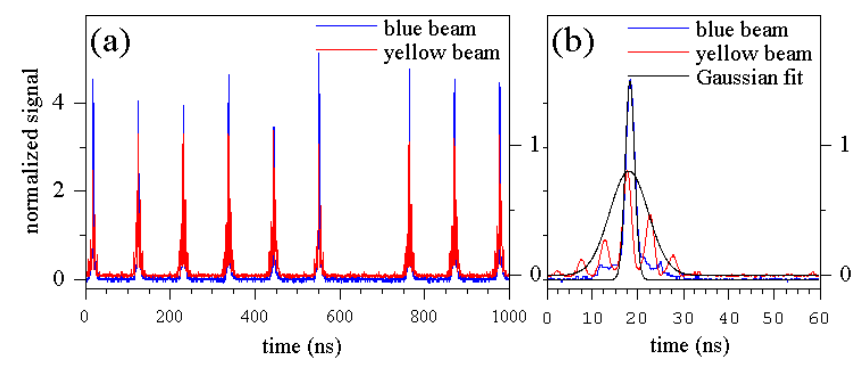

FIG. 18 (Color online) Longitudinal d-Au bunch, deuteron - blue beam, Au ion - yellow beam, RHIC beam fill \#9642. (a) A sub-section of the 95 bunch structure, showing the $106.6 \mathrm{~ns}$ bunch spacing, and (b) detail of the bunch shape, $2.3 \mathrm{~ns}$ FWHM for the deuteron and $\sim 10 \mathrm{~ns}$ FWHM for the envelope of the $\mathrm{Au}$ ion bunch. Each bunch contains $6.3 \times 10^{8} \mathrm{Au}$ ion and $9 \times 10^{10}$ deuterons both of $100 \mathrm{GeV}$ per nucleon.

\section{B. BEAM PROFILE OF 4.6 GeV GOLD IONS}

At the end of the Run- 8 beam program, two Au ion beams are injected in the RHIC ring but with a low energy of $4.6 \mathrm{GeV}$ per nucleon on each ring. Although the $\mathrm{Au}$ ion energy is less than the previous d-Au runs, the residual gas generates sufficient luminescence photon. Figure 19 displayed the luminescence image of both Au ion beams for RHIC beam fill \#10003. Again no interference band-pass filter is used, a much longer exposure time of 50 minutes is typically employed, and post image processing is performed on the raw luminescence images. A total of $3.5 \times 10^{18} \mathrm{Au}$ ions per beam passed through the interaction chamber resulted in a total of $\sim 1.7 \times 10^{11}$ photons emitted in a $4 \pi$ emission solid angle. Hence the photon production efficiency reaches $4.7 \times 10^{-8}$ photons/Au ion. This efficiency is slightly lower (by a factor of 2) than that of the higher energy Au ion beam.

$\begin{aligned} & \text { Because of the lower } \mathrm{Au} \text { ion beam energy, a } \\ & \text { beam scaled up by a factor of }\end{aligned}$
$\sqrt{100 \mathrm{GeV} / 4.6 \mathrm{GeV}}=4.7, \quad$ is anticipated.

Experimentally a beam size increase of 4.8 is observed which is in good agreement with prediction. The wide Au ion beams are clearly obscured by the front wires of the rf cage at the center of the chamber. Nonetheless, one can still determine their beam sizes from the image profile, see Figure 19. Beam sizes of $6.1 \mathrm{~mm}$ FWHM $(2.6 \mathrm{~mm} \sigma)$ and $5.4 \mathrm{~mm}$ FWHM (2.4 $\mathrm{mm} \sigma)$ are inferred for the yellow and blue $\mathrm{Au}$ ion beams, respectively. And they have $\sim 3.3 \sigma$ separation.

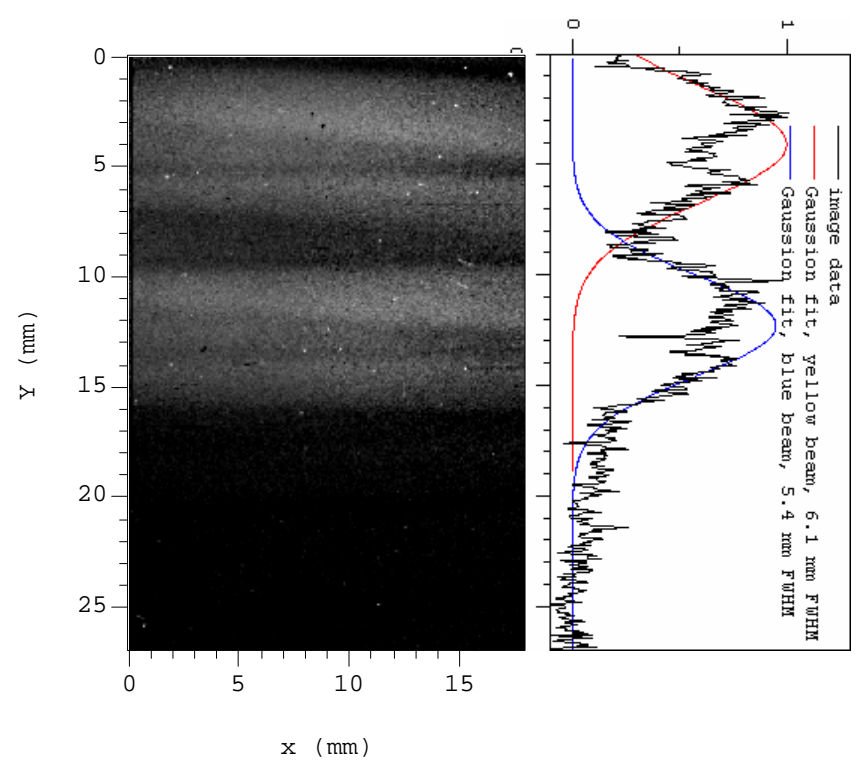

FIG. 19. (Color online) H-jet image and the Gaussian curve fit of a luminescence profile of an $\mathrm{Au}-\mathrm{Au}$ run for both RHIC rings. Top (bottom) yellow (blue) beam. No interference filter is used, RHIC beam fill \#10003, 60 bunches/ring, 3000-second exposure, total of $4 \times 10^{19} \mathrm{Au}$ ions/ring.

Beam emittance calculated from these beam sizes gives 19.8 and $15.5 \pi \cdot \mathrm{mm} \cdot \mathrm{mrad}$ for yellow and blue beams. While the RHIC run overview posted an average emittance of $40-60 \pi \cdot \mathrm{mm} \cdot \mathrm{mrad}$ for the Au-Au runs. Likewise, the corresponding IPM data shown in Figure 20 yields beam sizes of $13.7 \mathrm{~mm}$ FWHM (5.85 $\mathrm{mm} \sigma$ ) and $7.4 \mathrm{~mm}$ FWHM (3.15 $\mathrm{mm} \sigma$ ) for the yellow and blue $\mathrm{Au}$ ion beams, respectively. Calculated emittances of 15.6 and 4.09 $\pi \cdot \mathrm{mm} \cdot \mathrm{mrad}$ are derived using these IPM data. We note that during low energy $\mathrm{Au}-\mathrm{Au}$ runs the IPM data varies dramatically from different beam fills to different beam buckets resulting in dissimilar beam emittance values. On the other hand, the beam size and emittance obtained from the luminescence profile should be an absolute upper limit for the low energy $\mathrm{Au}$ ion beams. Table 3 listed the $\mathrm{Au}-\mathrm{Au}$ beam comparison between the IPM and the luminescence monitor.

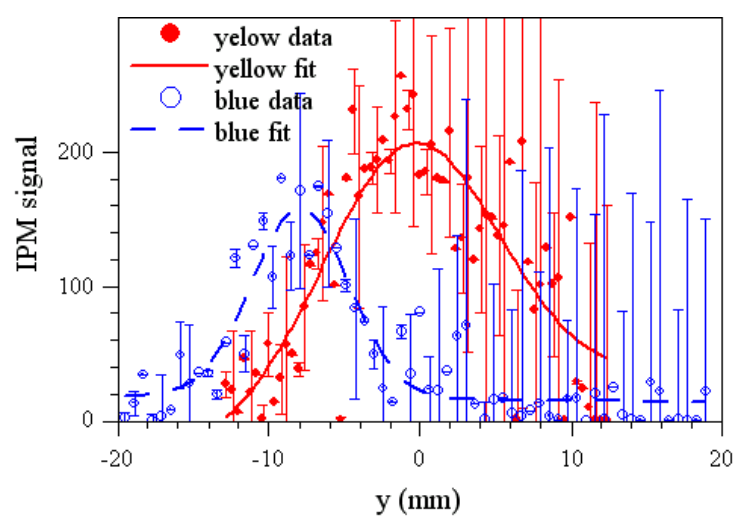


FIG. 20. (Color online) RHIC ring averaged vertical IPM data for fill \#10003 Au-Au run. A Gaussian curve fit to the data gives a blue beam width of $7.4 \mathrm{~mm}$ FWHM ( $3.15 \mathrm{~mm} \sigma)$ and yellow beam width of $13.75 \mathrm{~mm}$

Table 3 Comparison between H-jet and IPM beam profiles for d-Au and Au-Au runs. Only Au beam parameters are compared, deuteron (blue beam) parameters are not listed.

\begin{tabular}{|l|l|l|l|l|l|l|}
\hline RHIC run & \multicolumn{1}{|c|}{ d-Au run, fill \# 9642 } & \multicolumn{4}{c|}{ Au-Au run, fill \# 10003 } \\
\hline beam profiler & \multicolumn{1}{|c|}{ H-jet } & \multicolumn{1}{|c|}{ IPM } & \multicolumn{2}{c|}{ H-jet } & \multicolumn{2}{c|}{ IPM } \\
\hline RHIC beam & yellow Au & yellow Au & yellow & blue & yellow & blue \\
\hline$\sigma(\mathrm{mm})$ & 0.51 & 1.90 & 2.6 & 2.3 & 5.85 & 3.15 \\
\hline$\beta$ betatron function & 3 & 174 & 10 & 10 & 63.6 & 70.56 \\
\hline$\gamma$ function & 107.4 & 107.4 & 4.9 & 4.9 & 4.9 & 4.9 \\
\hline $\boldsymbol{\varepsilon}(\boldsymbol{\pi}$ mm mrad) & $\mathbf{5 5 . 8}$ & $\mathbf{1 3 . 5}$ & $\mathbf{1 9 . 8}$ & $\mathbf{1 5 . 5}$ & $\mathbf{1 5 . 6}$ & $\mathbf{4 . 0 9}$ \\
\hline center $(\mathrm{mm})$ & 11.3 & -4.86 & 4 & 12 & -0.58 & -7.92 \\
\hline beam separation & - & - & \multicolumn{2}{|c|}{$\sim 3.3 \sigma$} & & $\sim 1.6 \sigma$ \\
\hline
\end{tabular}

\section{SPECTRAL SIGNATURE OF AU ION BEAM INDUCED LUMINESCENCE}

The luminescence produced by the excitation of $100 \mathrm{GeV}$ Au ions impinge on residual gas is $\sim 2$ orders of magnitude lower than that of the polarized proton excitation on H-jet. The online fiber-optics spectrometer do not has sufficient sensitivity to capture its detail luminescence spectrum. Instead qualitative spectral information is inferred by spectrally filtering the images collected by the H-jet camera. Figure 21 shows four selected images to draw our initial conclusion on the spectral content of the residual gas. The fact that both images of $\mathrm{H}_{\alpha}(656 \mathrm{~nm})$ and $\mathrm{H}_{\alpha}(486 \mathrm{~nm})$ lines show a clear Au ion beam path and the relative intensity ratio of these two image agrees with expectation suggested that the residual gas in the chamber (in $10^{-9}$ Torr vacuum) contains hydrogen. However, when a near infrared (NIR) passed filter (RG9, 700-900 nm pass) is placed in front of the CCD camera, an unambiguous Au ion streak is also observed. This NIR light contains near $20 \%$ of the total luminescence intensity. Therefore, it is reasonable to conclude that hydrogen is still the main constitute of the residual gas for the production of Au ion induced luminescence. However, residual gas other than hydrogen also participates in this luminescence process. In order to clarify the content of the residual gas, it is necessary to install a residual gas analyzer at the center of the interaction chamber in future RHIC beam runs.
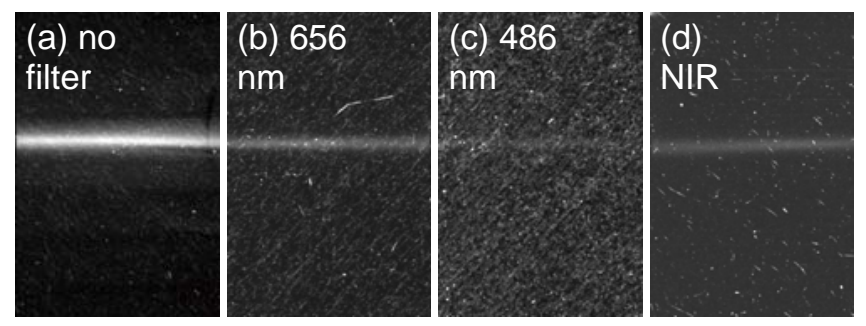

FIG. 21. Image of $\mathrm{Au}$ ion beam induced luminescence on residual gas after different interference band-pass filters, $100 \mathrm{GeV} \mathrm{d-Au} \mathrm{run.} \mathrm{All} \mathrm{images} \mathrm{are}$ acquired with 5 minutes of camera exposure. (a) no filter in front of the CCD, (b) $656 \mathrm{~nm}$ filter to select the $\mathrm{H}_{\alpha}$ emission line, (c) $486 \mathrm{~nm}$ filter to select the $\mathrm{H}_{\beta}$ emission line, and (d) a RG9 near infrared filter to select emission from 700 to $900 \mathrm{~nm}$. Their relative intensity ratio is 1:0.4:0.15:0.2, respectively.

The fact that hydrogen gas is still the main source of luminescence has some advantage on constructing beam profile monitors. Unlike many common gases, all known excited states of hydrogen has a relatively short decay lifetime of $<100 \mathrm{~ns}$. Therefore, no serious image smearing cause by gas diffusion is anticipated when long exposure time is needed.

\section{CONCLUSION}

It is interesting to note that the amount of luminescence light estimated from the experimental data gives $8.6 \times 10^{-9}$ photon/proton (656 nm photons) for the injected hydrogen gas jet and $2.7 \times 10^{-8}$ photon/Au-ion for the residual gas. Although the absolute amount of light collected is $\sim 1-2$ orders of magnitude lower with the Au ion excitation on the residual gas, its light production efficiency is actually slightly higher than proton excitation on atomic hydrogen gas jet. Despite the gas density of the $\mathrm{H}$-jet is $\sim 10^{5}$ higher than the residual gas density of a typical $10^{-9}$ Torr vacuum, light luminescence by Au ion beam appears more favorable. If this light production obey the $\mathrm{Z}^{2}$ law from the Bethe-Bloch equation, $\mathrm{Z}_{\mathrm{Au}}=79 \& \mathrm{Z}_{\mathrm{H}}=1, \mathrm{Au}$ ions beam would produce $6 \times 10^{3}$ more light than atomic hydrogen of the same beam energy and intensity. Our results are in consistent with this orders of magnitude estimation. However, a detail calculation of visible light fluorescence cross-sections for $100 \mathrm{GeV}$ protons and $\mathrm{Au}$ ions incident on atomic hydrogen at different gas density is needed to aid in the design of such future RHIC beam profile monitors.

Successful operation of the H-jet camera and the fiber-coupled spectrometer system offers an independent confirmation of the RHIC beam characteristics but complementary to the ionization beam profile monitor. Using particle beam induced fluorescence from injected gas, this optical beam diagnostic technique provides a simple measurement of beam 
characteristics. The H-jet luminescence camera system can give a near real-time guidance for RHIC beam tuning, injection, and finally at store. Not only a remarkable true image of the RHIC beam is in sight, various RHIC beam parameters for substantiating the emittance preservation along the RHIC accelerator are also derived. This is of vital important to the RHIC accelerator. Beam profile monitors based on the luminescence of injected or residual gas excited by the passage of particle beams is a promising approach to diagnosis low energy to high energy particle beams, including protons, ions, and possibly deuterons.

The images of Au ion beams of high and low energies are a clear signature of beam excitation and luminescence of residual gas. Although low energy particle beam induced light emission from hydrogen has been known for quite some time [12], our finding represents a substantial step towards the realization of a simple yet truly noninvasive beam monitor for highenergy particle beams with unprecedented clarity. To minimize ionization radiation damage to CCD and MCP by the particle beam, it is necessary to couple the luminescence light onto a radiation-hard coherent imaging fiber and have the camera system located away from the radiation environment. And by positioning 2 sets of imaging system orthogonal to the direction of the particle beam, a 2-dimensonal transverse beam monitor of high-energy particle beam can be realized. Together with a wall-current-monitor system and/or a low light level optical temporal measurement system, a 3-dimensional particle beam profile system can be envisioned providing routine diagnosis of high-energy particle beams.

\section{ACKNOWLEDGMENTS}

This work was supported by U.S. Department of Energy under Contract No. DE-AC02-98CH10886

\section{REFERENCE}

[1] N. Luciano, A. Nass, Y. Makdisi, P. Thieberger, D. Trbojevic, and A. Zelenski, Proc. of Particle Accelerator Conference (2005), p 4293.

[2] T. Russo, S. Bellavia, D. Gassner, P. Thieberger, D. Trbojevic, and T. Tsang, Proc. of Particle Accelerator Conference (2007), p 4648.

[3] visit http://www.bnl.gov/rhic/

[4] A. Zelenski, J. Alessi, A. Bravar, G. Bunce, M.A. Chapman, D. Graham, W. Haeberli, H. Hseuh, V. Klenov, H. Kolster, S. Kokhanovski, A. Kponou. V. Lodestro, W. MacKay, G. Mahler, Y. Makdisi, W. Meng, J. Ritter, T. Roser, E. Stephenson, T. Wise, and V. Zoubets, AIP Conf. Proc. V675, 954 (2003).

[5] A. Bravar, AIP Conf. Proc.V675, 830 (2003).

[6] A. Zelenski, A. Bravar, D. Graham, W. Haeberli, S. Kokhanovski, Y. Makdisi, G. Mahler, A. Nass, J. Ritter, T. Wise, and V. Zubets, Nucl. Instr. Methods A 536, 248 (2005).

[7] Y. Makdisi, I. Alekseev, S. Bellavia, A. Bravar, G. Bunce, M.A. Chapman, S. Dhawan , K.O. Eyser, D. Gasner, R. Gill , W. Haeberli, Z. Li, A. Khodinov, A. Kponou, W. Meng, A. Nass, H. Okada, N. Saito, S. Resica1, E. Stephenson, D. Svirida, D. Trbojevic, T. Tsang, T. Wise, A. Zelenski, V. Zubets, AIP Conf. Proc. V915, 975 (2007).

[8] D. Trbojevic, H.C. Hsueh, W. MacKay, A. Dress, and R. Fliler, Proc. of Particle Accelerator Conference (2001), p 3141.

[9] G. Burtin, J. Camas, G. Ferioli, R. Jung, J. Koopman, R. Perret, A. Variola, J. M Vouillot, Proc. of European Particle Accelerator Conference (2000), p.255.

[10] F. Becker, C. Andre, P. Forck, D.H.H. Hoffmann, and H. Iwase, , Proc. of European Particle Accelerator Conference (2006), p.1013.

[11] H. Okada, I. Alekseev, A. Bravar, G. Bunce, S. Dhawan, K.O. Eyser, R. Gill, W. Haeberli, H. Huang, O. Jinnouchi, Y. Makdisi, I. Nakagawa, A. Nass, N. Saito, E. Stephenson, D. Sviridia, T. Wise, J. Wood, and A. Zelenski, AIP Conf. Proc. V2, 1389 (2008).

[12] S. Fraser, IEEE Trans. Nucl. Science, Vol. NS-28, No.3, 2084 (1981). 